'Instituto de Ciencias Jurídicas, Universidad Austral de Chile. Puerto Montt, Chile.

IIndustrial and Management Systems Engineering, University of South Florida. Tampa, Florida, Estados Unidos. a Doctora en Derecho. ${ }^{\mathrm{b}} \mathrm{Ph} . \mathrm{D}$. in Industrial Engineering.

Financiamiento: Proyecto de investigación Fondecyt Regular №1211572.

Recibido el 11 de agosto de 2021, aceptado el 10 de septiembre de 2021

Correspondencia a:

Pamela Mendoza-Alonzo Los Pinos S/N, Balneario Pelluco, Puerto Montt, Chile. pamela.mendoza@uach.cl

\section{Telemedicina: desafíos para Chile a la luz de la experiencia de Estados Unidos durante la pandemia}

\author{
PAMELA MENDOZA-ALONZO ${ }^{1, a}, J^{2}$ NNIFER MENDOZA-ALONZO ${ }^{2, b}$
}

\section{Telemedicine: expected challenges in Chile based on the experience in the United States during the pandemic}

Telemedicine has had a significant role during the outbreak of COVID-19. The experience in the United States has shown advantages and some challenges that need to be addressed to include telemedicine as an established part of the health system. This article aims to determine the United States' main challenges, associating them with the Chilean reality. In this study, we classify the barriers and difficulties for telemedicine into three areas: accessibility, cyber security, and medical liability. We argue that Chile will have to deal with similar obstacles to include telemedicine as a regular health service for the entire population.

(Rev Med Chile 2021; 149: 1198-1204)

Key words: COVID-19; Cyber Security; Health Equity; Medical Liability; Telemedicine.

\section{Telemedicina en Estados Unidos}

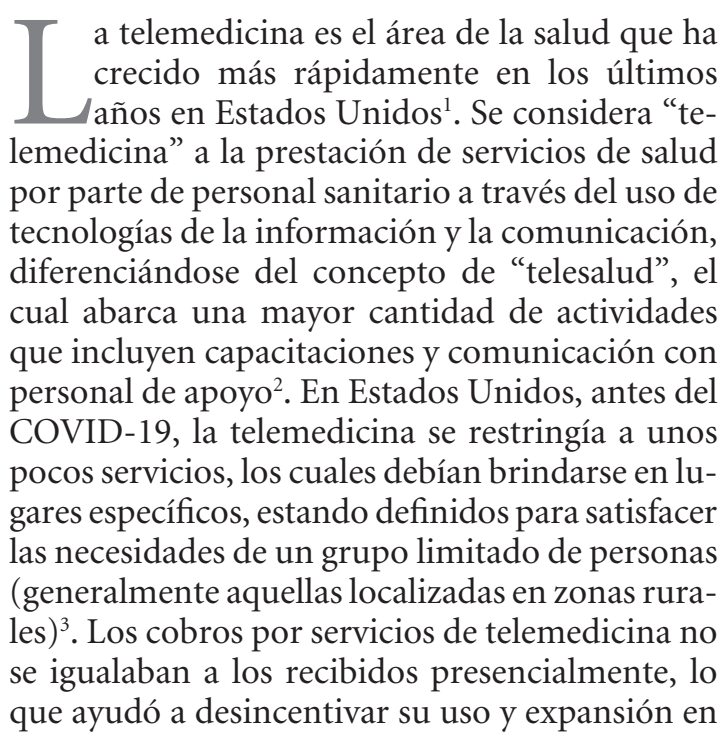

los centros de salud ${ }^{4}$. Las ventajas de la telemedicina llevaron a que en Estados Unidos se discutiera su mejora con anterioridad; no obstante, las leyes y reglamentos no se materializaron completamente sino hasta la aparición de los primeros casos de SARS-CoV-2 en el país 5 .

Considerando el estado de emergencia producto del COVID-19, los Centros de Servicios de Medicare y Medicaid (CMS por sus siglas en inglés) autorizaron de forma temporal la expansión de los servicios de telemedicina para sus beneficiarios en marzo del 2020 a través de la Ley de Apropiaciones Suplementarias de Preparación y Respuesta al Coronavirus ${ }^{6}$. Los CMS son una agencia federal de Estados Unidos que administran los principales programas de salud que benefician mayoritariamente a adultos mayores y personas de bajos recursos del país ${ }^{7}$. La expansión en los servicios administrados y el aumento en los reembolsos (ahora 
equiparados a la atención presencial) vinieron a atenuar los bajos ingresos de los centros de salud (especialmente de consultas pequeñas), a brindar una alternativa segura a los pacientes más vulnerables y a ayudar a contener el virus ${ }^{8}$. Posteriormente, los seguros de salud privados se unieron, aprobando y flexibilizando los servicios y cobros por telemedicina'. Por su parte, la Oficina de Derechos Civiles (OCR, por sus siglas en inglés) emitió, previo a la flexibilización de los servicios de telemedicina por parte de los CMS, la Notificación de Discreción de Cumplimiento de la Ley de Portabilidad y Responsabilidad de Seguros Médicos (HIPAA por sus siglas en inglés) permitiendo excepcionalmente, por ejemplo, la utilización de plataformas de uso común para proveer servicios de telemedicina ${ }^{10}$.

La razón de legislar por parte de Estados Unidos en torno a los servicios y reembolsos de telemedicina radica en los beneficios que esta trae para el paciente, personal sanitario y el sistema de salud en general, los cuales trascienden el estado de emergencia de salud pública actual $^{11}$. Los pacientes ahorran tiempo y dinero en transporte, disminuyendo ausencias a las citas médicas, especialmente aquellos pacientes de zonas aisladas, mejorando los resultados en salud ${ }^{12}$. Además, la telemedicina reduce el riesgo de contraer infecciones en personas ancianas y pacientes inmunodeficientes ${ }^{11,13}$. Al igual que el cuidado en la casa, la telemedicina ayuda al personal médico a identificar aspectos claves del paciente, por ejemplo, condiciones de vivienda, salubridad y alimentación, permitiendo brindar cuidados más personalizados, centrados en el paciente $^{14}$. Igualmente, los proveedores de salud pueden abarcar un número mayor de pacientes, mejorando sus ingresos, considerando que la telemedicina disminuye los costos por servicio entregado ${ }^{15}$. No obstante, la telemedicina es aún una prestación de cuidados que requiere de especiales consideraciones en su implementación. Si bien la pandemia ha acelerado su uso en varios países del mundo, incluyendo Chile, hay varios aspectos que deben ser abordados si se desea incorporar los servicios de telemedicina de forma permanente al sistema de salud. Estos aspectos, motivo de discusión en Estados Unidos, son resumidos en el siguiente apartado. Los alcances en el escenario chileno son también analizados en el presente artículo.

\section{Barreras y retos de la telemedicina en Estados Unidos}

\section{Accesibilidad}

Más de 21 millones de personas no tienen acceso a internet en Estados Unidos, lo que limita el alcance de los servicios de telemedicina a toda la población ${ }^{16}$. Esto ha derivado a que se discuta la incorporación de "acceso a banda ancha" como un nuevo determinante social de salud ${ }^{17}$, dado que las personas que necesitan mayor asistencia sanitaria (adultos mayores y personas de escasos recursos) son las que además presentan más barreras digitales $^{18}$. En efecto, en un estudio realizado a más de 600 mil beneficiarios de Medicare (programa de seguro de salud estadounidense esencialmente para adultos mayores) expuso que, en promedio, $41,4 \%$ de ellos no tiene acceso a un computador e internet de alta velocidad y $40,9 \%$ no tiene un teléfono inteligente con internet ${ }^{18}$. Estas cifras incrementan si se consideran personas de más de 85 años, viudos o viudas y con baja escolaridad ${ }^{18}$. La brecha tecnológica genera alarma en Estados Unidos dado que pudiese ser un factor que, a través de la telemedicina, amplíe aún más la ya existente inequidad en el acceso a la salud ${ }^{19}$, perjudicando especialmente a personas de zonas rurales, de bajos ingresos, adultos mayores y minorías raciales y étnicas ${ }^{17,20}$.

A la brecha digital se suman también las dificultades relativas al acceso de personas con ciertos tipos de discapacidades que requieren de tecnologías específicas para una correcta comunicación ${ }^{21}$. Annaswamy y cols. ${ }^{22}$ manifiestan que las plataformas de telemedicina utilizadas actualmente en Estados Unidos no cuentan con interfaces adecuadas para facilitar la comunicación con personas ciegas, sordas o con problemas cognitivos. Lam y cols. ${ }^{23}$ estiman que en Estados Unidos 38\% de los adultos mayores de 65 años o más y $72 \%$ de personas sobre los 85 años presentan dificultades físicas (problemas visuales, sordera) o cognitivas (problemas para expresarse, demencia), imposibilitando el contacto adecuado al momento de recibir servicios de telemedicina. A pesar de que Estados Unidos cuenta con la Ley para Estadounidenses con Discapacidades (ADA por sus siglas en inglés) desde 1990, esta Ley no contempla estándares de accesibilidad específicos para el contexto virtual ${ }^{22}$.

Si bien las barreras financieras aplicadas a 
los servicios de telemedicina se han relajado a propósito del COVID-19 permitiendo un acceso masivo a la telemedicina, no se tiene certeza de cómo operarán los reembolsos por estos servicios post pandemia ${ }^{3}$. Esto se debe a que, por ejemplo, los pagos por atención a través de videollamadas se han equiparado a las realizadas con solo audio. Sin embargo, los servicios de telemedicina vía teléfono no se consideran apropiados cuando se requiere evaluación visual del paciente ${ }^{18}$. En general, la regulación no es uniforme y solo en algunos estados los pagos por servicios de telemedicina son similares a los pagos por servicios presenciales (a través de las llamadas parity laws) ${ }^{3}$. Por regla general, el reembolso es menor cuando se trata de telemedicina, por consiguiente, si se retoman los pagos existentes prepandemia, persistirá el desincentivo a su uso e implementación ${ }^{24,3}$.

\section{Ciberseguridad}

La flexibilización en las restricciones a la telemedicina impuestas en Estados Unidos incrementó su uso en $154 \%$ en marzo de 2020 comparado con el mismo mes de $2019^{25}$. En los meses posteriores, se estimó que alrededor de $30 \%$ de las visitas a los centros de salud se realizaron a través de telemedicina ${ }^{26}$. Este incremento constante y rápido en el uso de esta modalidad de servicio durante la pandemia ha traído también un aumento en los riesgos de ataques cibernéticos al sector salud, especialmente a hospitales ${ }^{27}$. Asimismo, se advierte que las conexiones desde la casa son riesgosas dado que no cuentan con la seguridad necesaria para resguardar la confidencialidad en la comunicación, protección de los datos y privacidad de los pacientes ${ }^{28,29}$. Entre los ejemplos de estos ataques cibernéticos a los pacientes se encuentran la intercepción de datos, ataques a conexiones intermedias entre el paciente y el sistema de telemedicina, amenazas por instrumentos perdidos o claves de seguridad débiles, alteraciones en las prescripciones $^{28}$. Los servicios de telemedicina deben ser provistos bajo estructuras tecnológicas adecuadas tales como; encriptación de datos, uso de antivirus, actualización constante de los software y uso de autenticación de múltiples factores, entre otros recursos de seguridad ${ }^{30}$.

A través de la Ley federal HIPAA de 1996, Estados Unidos ha fijado estándares de seguridad y protección a la privacidad del paciente, los que se aplican también para los servicios de telemedi- cina. En especial, la HIPAA Privacy Rule regula la protección del historial médico (creación, mantenimiento y posterior destrucción) así como el manejo de otros datos sensibles de los pacientes ${ }^{31}$. En consecuencia, como no hay mayores diferencias en la práctica con la medicina presencial ${ }^{32}$, se debe contar con una ficha médica (electrónica en este caso) que incluya copia de correos electrónicos, recetas, exámenes, consentimiento informado, entre otros, la cual debe estar debidamente resguardada y accesible tanto para al médico como para el paciente $^{33}$. Con todo, la velocidad de propagación del COVID-19 ha impedido que todos los centros de salud implementen los servicios de telemedicina acorde a estas normas. Por ello, la OCR estableció que: "ejercerá su discreción de cumplimiento y no les impondrá sanciones por el incumplimiento de los requisitos reglamentarios de las Normas de la ley HIPAA a los proveedores de atención médica cubiertos, en relación con la prestación de servicios de telemedicina de buena fe durante la emergencia nacional de salud pública por el COVID-19"10. Entre las medidas de flexibilización se ha permitido la atención a través de plataformas de uso común (WhatsApp, Skype, MS Teams o Zoom) ${ }^{10}$, advirtiendo que se deben tomar todas las medidas de encriptación y privacidad posibles para prever los riesgos de su uso ${ }^{10}$ dado que estas plataformas están más expuestas a problemas de ciberseguridad ${ }^{30}$.

Según un estudio realizado en Massachusetts en el 2020 a prestadores de servicios de salud, éstos consideran que, incluso si los problemas relativos a ciberseguridad estuviesen resueltos, no se puede brindar una atención adecuada haciendo solo uso de los servicios de telemedicina (aunque también concuerdan en que la telemedicina debería seguir siendo parte de las formas de proveer cuidado ${ }^{34}$. El uso de la telemedicina dificulta, entre otros aspectos, la correcta ejecución de protocolos que incluyen privacidad a la hora de discutir aspectos complejos y brindar soporte emocional al paciente cuando lo necesita. Un claro ejemplo ocurre en la oncología, en la que existen estrictos protocolos para dar noticias difíciles a los pacientes (los más usados son los six steps SPIKES protocol), que requieren interacción física para su debida aplicación ${ }^{35}$.

\section{Responsabilidad médica}

En Estados Unidos se requiere de una licencia profesional para ejercer en cada estado, agregando 
otro obstáculo al desarrollo de la telemedicina ${ }^{36}$. El personal de salud debe tomar los resguardos necesarios para confirmar que el paciente se encuentra localizado en el estado en que el médico tratante tiene licencia ${ }^{37}$, y así no arriesgar sanciones por ejercicio ilegal de la profesión ${ }^{33}$. Por otro lado, aunque existe una tendencia (incluso anterior a la pandemia) de otorgar licencias interestatales ${ }^{38}$, para esos casos se debe tener en consideración que el médico se somete a más de una jurisdicción y, por ende, puede ser condenado por sus eventuales responsabilidades en más de un estado ${ }^{36}$. En consecuencia, tanto el médico como el resto del personal de salud que preste servicios en la modalidad de telemedicina deberán tener en cuenta el contexto de cada estado en particular ${ }^{36}$.

De igual modo, las limitaciones que impone la distancia física en la telemedicina incrementan el riesgo de incurrir en la denominada medical professional liability, por errores de diagnóstico o diagnósticos tardíos de enfermedades graves, falta de seguimiento a procedimientos o enfermedades, prescripción errada de medicamentos, entre otros $^{36}$. Para mitigar estos riesgos, es necesario que el paciente suscriba un consentimiento informado especial que incluya las particularidades de la atención a distancia y que se adapte a la regulación de cada estado ${ }^{32,36}$. La misma precaución se debe tener con los seguros de responsabilidad (que suelen tener una cobertura restringida a los límites del estado $)^{33,37,39}$, y comprobar que el médico tratante se encuentre cubierto en el ejercicio de la telemedicina tanto en el estado en que reside como en los otros en los que está autorizado para ejercer ${ }^{32}$.

\section{Presente y futuro de la telemedicina en Chile}

El desarrollo de la telemedicina en Chile no es tan distinto al observado en Estados Unidos. Si bien los primeros indicios se remontan al año 1993 (con el Proyecto Piloto Universidad Católica/ Hospital Dr. Sótero del Río ${ }^{40}$, la expansión de la telemedicina también ha sido consecuencia inmediata de la pandemia. Asimismo, tampoco existe una regulación uniforme y específica, por lo que se aplican principalmente reglas generales ${ }^{41}$. Dentro de este marco normativo general, la Ley $\mathrm{N}^{\circ} 20.584$ que regula los derechos y deberes de las personas en relación con su atención en salud establece el derecho de información, el derecho de la reserva de la información contenida en la ficha clínica y el derecho de la autonomía de las personas en su atención de salud (esta última manifestada a través del consentimiento informado), entre otros. Dicha ley se complementa con la Ley $\mathrm{N}^{\circ} 19.628$ sobre protección de la vida privada que considera como dato sensible "los estados de salud físicos o psíquicos". Como consecuencia de la pandemia, en marzo de 2020 se ingresó a tramitación un proyecto de ley (Boletín 13375-11) que autoriza a los prestadores de salud efectuar atenciones mediante telemedicina, a través de la modificación de la Ley No20.584. Por su parte, en el 2020 el Ministerio de Salud emitió una serie de resoluciones que autorizan la utilización transitoria de códigos del Fondo Nacional de Salud (FONASA) (homologable a las Instituciones de Salud Previsional) para diversas atenciones de telemedicina ${ }^{41}$. Esto se refuerza con la Resolución Exenta No220/2021 que incorpora más de 50 nuevos códigos de teleconsulta, reduciéndose con ello los posibles problemas de cobertura. Además, en septiembre de 2020 entró en vigor la Ley No21.267 que establece medidas para facilitar la adquisición de remedios en el contexto de una alerta sanitaria por epidemia o pandemia.

Sin embargo, Chile no cuenta con una ley equivalente a la HIPAA que fije altos estándares para resguardar la privacidad, protección de los datos y confidencialidad del paciente. En efecto, las recomendaciones que se han hecho para Chile sugieren tener en cuenta los estándares de seguridad de la HIPAA y otras leyes extranjeras a la hora de implementar la telemedicina ${ }^{42}$, dado que las plataformas utilizadas en Chile durante la pandemia no garantizan una adecuada protección de los datos del paciente. Por esta razón, el Centro Nacional en Sistemas de Información en Salud, con la colaboración de FONASA, ha implementado en el 2021 un "sello de calidad de software en telemedicina", el que opera bajo la norma internacional ISO 25010 y que se espera se generalice una vez terminada la pandemia ${ }^{43}$.

Sin perjuicio de las medidas adoptadas para facilitar la implementación de la telemedicina en Chile, la brecha y el analfabetismo digital son una realidad en el país. Aunque hoy en día el acceso a internet y tecnologías ha aumentado considerablemente, Cortés y cols. ${ }^{44}$ advierten que en Chile "el uso menos frecuente y variado de Internet se asocia a un menor nivel educativo, mayor edad, menor nivel socioeconómico y a ser mujer". Al 
igual que en Estados Unidos, esta es una realidad que preocupa, particularmente en el caso de los adultos mayores, quienes son un sector de la población que necesita mayores cuidados. Un estudio realizado en el 2020 a personas nacidas entre 1943 y 1958 de dos ciudades chilenas, Talca y San Joaquín, arrojó como resultado que $44 \%$ y $46,9 \%$, respectivamente, no tiene acceso a internet, y $67,5 \%$ en Talca y $60,3 \%$ en San Joaquín no sabe ocupar un computador ${ }^{45}$. Es necesario, por lo tanto, implementar programas de teleeducación dirigidos a la comunidad, para familiarizarla con las tecnologías de la información y la comunicación, y así acercar la telemedicina a las personas más vulnerables ${ }^{40}$.

Referente al ejercicio de la profesión, y a diferencia de Estados Unidos, no se requiere una licencia especial para ejercer la telemedicina a lo largo del territorio chileno. No obstante, se asemejan en que ante la ausencia de normas específicas que regulen la responsabilidad médica, se debe recurrir al derecho común donde los estándares exigidos y principios aplicables son los mismos a una atención presencial ${ }^{41}$. Sin perjuicio de ello y teniendo en cuenta los riesgos asociados a la atención a distancia, la Superintendencia de Salud a través de la Circular Nº7/2020 ha informado los deberes específicos que asisten a los prestadores individuales e institucionales de salud que otorgan atenciones remotas. Esto permite determinar eventuales responsabilidades en el caso que aquellos deberes no se observen y así aplicar adecuadamente la Ley No20.584. Debido al incremento de este tipo de prestaciones, es probable que aumenten también las demandas por mala praxis, planteando un desafío a los tribunales de justicia. Hasta el momento los tribunales no han tenido que pronunciarse expresamente, destacando solo la sentencia de 8 de noviembre de 2018 ( $R o l$ 24742-2018) de la Corte de Apelaciones de Santiago. La Corte acogió el recurso de protección interpuesto por una paciente con posible patología del Plan de Acceso Universal a Garantías Explícitas quien solicitó una atención presencial con un especialista, dado que no se llevó a cabo una atención adecuada a través de telemedicina ${ }^{46}$. Para evitar la judicialización es fundamental que los prestadores de salud soliciten un consentimiento informado a los pacientes que dé cuenta de las particularidades de la atención remota ${ }^{47}$, asegurándoles una atención equivalente a la presencial, con el debido resguardo de sus datos sensibles, confidencialidad y privacidad ${ }^{41}$.

\section{Referencias}

1. American Hospital Association. Health Industry Cybersecurity - Securing Telehealth and Telemedicine [En línea] 2021. Disponible en: https://www.aha.org/system/ files/media/file/2021/04/health-industry-cybersecurity-securing-telehealth-and-telemedicin-april-2021.pdf. [Último acceso: 4 de agosto de 2021].

2. Brackney J, Hulkower R, Pepin D, McCord R. Telehealth and telemedicine: a research anthology of law and policy resources. [En línea] Julio 2019. Disponible en: https:// www.cdc.gov/phlp/publications/topic/anthologies/anthologies-telehealth.html. [Último acceso: 24 de junio de 2021].

3. Bajowala S, Milosch J, Bansal C. Telemedicine pays: billing and coding update. Current Allergy and Asthma Reports 2020; 20 (10): 1-9.

4. Center for Connected Health Policy. State telehealth laws and reimbursement policies: a comprehensive scan of the 50 states \& the District of Columbia. The National Telehealth Policy Resource Cente. [En línea] Abril 2021. Disponible en: https://www.cchpca.org/ resources/state-telehealth-laws-and-reimbursement-policies-report-spring-2021/. [Último acceso: 03 de agosto de 2021].

5. Rockwell K, Gilroy A. Incorporating telemedicine as part of COVID-19 outbreak response systems. The American Journal of Managed Care 2020; 26 (4): 147-8.

6. U.S. Government Publishing Office. Coronavirus Preparedness and Response Supplemental Appropriations Act on March 6, 2020 [En línea]. Disponible en: https:// www.congress.gov/116/plaws/publ123/PLAW-116publ123.pdf. [Último acceso: 19 de julio de 2021].

7. Centers for Medicare \& Medicaid Services, 2021. [En línea]. Disponible en: https://www.cms.gov/About-CMS/ About-CMS. [Último acceso: 3 de agosto de 2021].

8. Centers for Medicare \& Medicaid Services. Medicare telemedicine health care provider fact sheet. 17 Marzo 2020. [En línea]. Disponible en: https://www.cms.gov/ newsroom/fact-sheets/medicare-telemedicine-health-care-provider-fact-sheet. [Último acceso: 24 de junio de 2021].

9. Verma S. Early impact of CMS expansion of Medicare telehealth during COVID-19. Health Affairs Blog. 15 Julio 2020. [En línea]. Disponible en: https://www. healthaffairs.org/do/10.1377/hblog20200715.454789/ full/. [Último acceso: 4 de agosto de 2021]. 
10. Office for Civil Rights. Notification of Enforcement Discretion for Telehealth Remote Communications During the COVID-19 Nationwide Public Health Emergency. 20 Enero 2021. [En línea]. Disponible en: https:// www.hhs.gov/sites/default/files/notificacion-de-discrecion-para-telemedicina.pdf. [Último acceso: 03 de agosto de 2021].

11. Seivert S, Badowski M. The Rise of Telemedicine: Lessons from a Global Pandemic. EMJ Innovations 2021; 5 (1): 64-9.

12. Drerup B, Espenschied J, Wiedemer J, Hamilton L. Reduced No-Show Rates and Sustained Patient Satisfaction of Telehealth During the COVID-19 Pandemic. Telemedicine and e-Health. 2021.

13. Portnoy J, Waller M, Elliott T. Telemedicine in the era of COVID-19. The Journal of Allergy and Clinical Immunology 2020; 8 (5): 1489-91.

14. Hasselfed B. Benefits of Telemedicine. John Hopkins Medicine 2020. [En línea]. Disponible en: https://www. hopkinsmedicine.org/ healthtreatment-tests-and-therapies/benefits-of-telemedicine. [Último acceso: 4 de agosto de 2021].

15. Antoniotti N. Business Aspects of Telemedicine. Telemedicine, Telehealth and Telepresence, Springer Cham 2021; 141-55.

16. Eruchalu N, Pichardo M, Bharadwaj M, Rodríguez C, Rodríguez J, Bergmark R, et al. The expanding digital divide: digital health access inequities during the COVID-19 pandemic in New York City. Journal of Urban Health. 2020; 98 (2): 183-6.

17. Fridsma D. American Medical Informatics Association (AMIA) response to FCC notice on accelerating broadband health tech availability. 24 Mayo 2017. [En línea]. Disponible en: https://www.amia.org/sites/default/ files/AMIA-Response-to-FCC-Notice-on-Accelerating-Broadband-Health-Tech-Availability.pdf. [Último acceso: 2 de agosto de 2021].

18. Roberts E, Mehrotra A. Assessment of disparities in digital access among Medicare beneficiaries and implications for telemedicine. JAMA Internal Medicine 2020; 180 (10): 1386-9.

19. Hero J, Zaslavsky A, Blendon R. The United States leads other nations in differences by income in perceptions of health and health care. Health Affairs 2017; 36 (6): 1032-40.

20. Nouri S, Khoong C, Lyles R, Karliner L. Addressing equity in telemedicine for chronic disease management during the Covid-19 pandemic. NEJM Catalyst Innovations in Care Delivery 2020; 1 (3).

21. Zhai Y. A call for addressing barriers to telemedicine: health disparities during the COVID-19 pandemic,
Psychotherapy and Psychosomatics 2020; 1.

22. Annaswamy T, Verduzco-Gutierrez M, Frieden L. Telemedicine barriers and challenges for persons with disabilities: Covid-19 and beyond. Disability and Health Journal 2020; 13 (4): 100973.

23. Lam K, Lu A, Shi Y, Covinsky K. Assessing telemedicine unreadiness among older adults in the United States during the COVID-19 pandemic. JAMA internal medicine 2020; 180 (10): 1389-91.

24. Baumann B, MacArthur K y Michalski J. The importance of temporary telehealth parity Laws to improve public health during COVID-19 and future pandemics. International Journal of Radiation Oncology, Biology, Physics 2020; 108 (2): 362.

25. Koonin L, Hoots B, Tsang C, Leroy Z, Farris K, Jolly $\mathrm{B}$, et al. Trends in the use of telehealth during the emergence of the COVID-19 pandemic-United States, January-March 2020. Morbidity and Mortality Weekly Report 2020; 69 (43): 1595.

26. Demeke H, Marks S, Pao L, Romero L, Sandhu P, Clark H. Trends in Use of Telehealth Among Health Centers During the COVID-19 Pandemic-United States, June 26-November 6, 2020. Morbidity and Mortality Weekly Report 2021; 70 (7): 240.

27. NJCCIC Advisory. Cyber Threats \& Cybersecurity for Healthcare During COVID-19, 04 Agosto 2020. [En línea]. Disponible en: https://www.cyber.nj.gov/ alerts-advisories/cyber-threats-cybersecurity-for-healthcare-during-covid-19. [Último acceso: 04 de agosto de 2021].

28. Lidong W, Cheryl A. Cyber security during the COVID-19 pandemic. AIMS Electronics and Electrical Engineering 2021; 5 (2): 146-57.

29. Gillespie S, Steven M, Bardakh A. Innovation through regulation: COVID-19 and the evolving utility of telemedicine. Journal of the American Medical Directors Association 2020; 21 (8): 1007-9.

30. Jalali M, Landman A, Gordon W. Telemedicine, privacy, and information security in the age of COVID-19. Journal of the American Medical Informatics Association 2021; 28 (3): 671-2.

31. U.S Department of Health \& Human Services. The HIPAA Privacy Rule. 10 Diciembre 2020. [En línea]. Disponible en: https://www.hhs.gov/hipaa/for-professionals/privacy/index.html. [Último acceso: 4 de agosto de 2021].

32. Fields B. Regulatory, legal, and ethical considerations of telemedicine. Sleep Medicine Clinics 2020; 15 (3): 409 16.

33. Kmucha S. Telemedicine and Physician Liability Issues. Ear, Nose \& Throat Journal 2020. 
34. Vosburg R, Robinson K. Telemedicine in Primary Care During the COVID-19 Pandemic: Provider and Patient Satisfaction Examined, Telemedicine and e-Health. 2021.

35. Wolf I, Waissengrin B, Pelles S. Breaking bad news via telemedicine: A new challenge at times of an epidemic. The oncologist 2020; 25 (6): e879-80.

36. George G, Brandon H, Legal and Regulatory Implications of Telemedicine, Springer, Cham 2021.

37. Bruhn H. Telemedicine: dos and don'ts to mitigate liability risk. Journal of American Association for Pediatric Ophthalmology and Strabismus 2020; 24 (4): 195-6.

38. American Telemedicine Association. State of Compacts. Mayo 2021. [En línea]. Disponible en: https://www.americantelemed.org/resources/state-of-compacts/ [Último acceso: 4 de agosto de 2021].

39. Hoffman D. Increasing access to care: telehealth during COVID-19. Journal of Law and the Biosciences 2020; 7 (1).

40. Subsecretaría de Redes Asistenciales. Programa Nacional de Telesalud: En el contexto de Redes Integradas de Servicios de Salud. 2018. [En línea]. Disponible en: http:// biblioteca.digital.gob.cl/handle/123456789/3635[Último acceso: 4 de agosto de 2021].

41. VV. AA. Lineamientos para el desarrollo de la telemedicina y telesalud en Chile. 1 Diciembre 2020. [En línea]. Disponible en: https://ineamientostelesalud.cl/2021/01/ segunda-edicion-de-los-fundamentos-para-los-lineamientos-para-la-telemedicina-y-telesalud-en-chile/
[Último acceso: 4 de agosto de 2021].

42. CENS. Guía de buenas prácticas y recomendaciones en telemedicina durante epidemia de COVID-19 en Chile. 14 April 2020. [En línea]. Disponible en: https://cens.cl/ guia-buenas-practicas-telemedicina/ [Último acceso: 4 de agosto de 2021].

43. CENS. Sello de Calidad. Evaluación de Calidad de Sistemas Software en Telemedicina. 14 Enero 2021. [En línea]. Disponible en: https://cens.cl/soluciones/sellos/ calidad-software-telemedicina/ [Último acceso: 4 de agosto de 2021].

44. Cortés F, De Tezanos-Pinto P, Helsper E, Lay S, Manzi J, Novoa C. ¿Se ha reducido la brecha digital en Chile? Diferencias entre acceso, uso y factores asociados al empleo de Internet. Midevidencias 2020; 22: 1-6.

45. Riveros C, Arenas A, Castro M, Olivares M. Políticas públicas, brechas y alfabetización digital de la persona mayor: la realidad chilena mirada desde las comunas de Talca y San Joaquín. Revista de Direito, Estado e Telecomunicações 2020; 12 (1): 137-58.

46. Domínguez C, Rubio F. Algunas consideraciones sobre telemedicina y responsabilidad civil. El Mercurio Legal, 11 Junio 2020. [En línea]. Disponible en: https://www. elmercurio.com/Legal/Noticias/Opinion/2020/06/11/ Algunas-consideraciones-sobre-telemedicina-y-responsabilidad-civil.aspx [Último acceso: 4 de agosto de 2021].

47. Mesa M, Pérez I. El acto médico en la era de la telemedicina. Revista Med Chile 2020; 148 (6): 852-7. 